\title{
Article \\ On a New Geometric Constant Related to the Euler-Lagrange Type Identity in Banach Spaces
}

\author{
Qi Liu ${ }^{+}(\mathbb{D})$ and Yongjin $\mathrm{Li}^{*,+}$ \\ School of Mathematics, Sun Yat-Sen University, Guangzhou 510275, China; liuq325@mail2.sysu.edu.cn \\ * Correspondence: stslyj@mail.sysu.edu.cn \\ t These authors contributed equally to this work.
}

check for updates

Citation: Liu, Q.; Li, Y. On a New Geometric Constant Related to the Euler-Lagrange Type Identity in Banach Spaces. Mathematics 2021, 9, 116. https://doi.org/10.3390/ math9020116

Received: 18 December 2020 Accepted: 5 January 2021 Published: 7 January 2021

Publisher's Note: MDPI stays neutral with regard to jurisdictional clai$\mathrm{ms}$ in published maps and institutional affiliations.

Copyright: (C) 2021 by the authors. Licensee MDPI, Basel, Switzerland. This article is an open access article distributed under the terms and conditions of the Creative Commons Attribution (CC BY) license (https:// creativecommons.org/licenses/by/ $4.0 /)$.

\begin{abstract}
In this paper, we will introduce a new geometric constant $L_{Y \mathrm{~J}}(\lambda, \mu, X)$ based on an equivalent characterization of inner product space, which was proposed by Moslehian and Rassias. We first discuss some equivalent forms of the proposed constant. Next, a characterization of uniformly non-square is given. Moreover, some sufficient conditions which imply weak normal structure are presented. Finally, we obtain some relationship between the other well-known geometric constants and $L_{Y J}(\lambda, \mu, X)$. Also, this new coefficient is computed for $X$ being concrete space.
\end{abstract}

Keywords: uniformly non-square Banach space; von Neumann-Jordan constant; Euler-Lagrange type identity

MSC: 46B20

\section{Introduction}

In the current decade, numerous geometric constants have been investigated for a Banach space $X$. Particular attention was given to the two constants; the von NeumannJordan constant $C_{\mathrm{NJ}}(X)$ and $J(X)$ (the James constant), where the results are rigorously investigated and analyzed. For a Banach space $X$, several studies on the James constant $J(X)$ and also on the von Neumann-Jordan constant $C_{\mathrm{NJ}}(X)$ have been conducted by Gao $[1,2]$, Yang and Wang [3], and Kato, Maligranda and Takahashi [4,5]. Interested readers in this field are advised to see the work presented in [6-11] and the references mentioned therein.

In the literature, there are many characterizations of inner product spaces. If we consider the usual Euclidean space $\left(R^{n},\|\cdot\|\right)$, the well-known identity $\|x+y\|^{2}+\| x-$ $y\left\|^{2}=2\right\| x\left\|^{2}+2\right\| y \|^{2}$ is called the parallelogram law. This identity can be extended to more general situations in several ways. Many authors have studied the necessary and sufficient conditions for a normed space to be an inner product space (for more details see, e.g., [12-14]).

Moslehian and Rassias [15] have recently proved the new equivalent characterization of inner product space using an Euler-Lagrange type identity which provide generalizations of parallelogram law. The result is presented in Section 3. The follow-up study of corresponding results of Moslehian and Rassias can be found in [16,17].

Motivated by the new characterization of inner product spaces by Moslehian and Rassias, we introduce a new geometric constant $L_{Y \mathrm{~J}}(\lambda, \mu, X)$ in a Banach space $X$. Some properties of this geometric constant are discussed.

The article is organized in the following way: we recall some fundamental concepts i.e., basic definitions with related axioms in the next section. In Section 3 some equivalent forms of $L_{Y J}(\lambda, \mu, X)$ and its relation to uniformly non-square are considered. Furthermore, we establish a new necessary condition for weak normal Banach spaces in the form of $L_{Y \mathrm{~J}}(\lambda, \mu, X)$. Section 4 is devoted to relationships between the constants $L_{Y \mathrm{~J}}(\lambda, \mu, X)$ and $C_{\mathrm{NJ}}(X)$, emphasized in terms of nontrivial inequalities involving these constants. Four 
illustrative examples are given as well. Finally, in the last section, we summarize the whole paper and put forward some questions.

\section{Preliminaries}

We pass now to introduce some notations. Let $X=(X,\|\cdot\|)$ be a real Banach space with $\operatorname{dim} X \geqslant 2, B_{X}=\{x \in X:\|x\| \leqslant 1\}$ its unit ball and $S_{X}=\{x \in X:\|x\|=1\}$ its unit sphere.

Recall that the Banach space $X$ is called uniformly non-square [18] if there exists a $\delta \in(0,1)$ such that for any $x, y \in S_{X}$ either $\frac{\|x+y\|}{2} \leq 1-\delta$ or $\frac{\|x-y\|}{2} \leq 1-\delta$. The constant

$$
J(X)=\sup \left\{\min \{\|x+y\|,\|x-y\|\}: x, y \in S_{X}\right\}
$$

is called the non-square or James constant of $X$.

The von Neumann-Jordan constant $C_{\mathrm{NJ}}(X)$ was defined in 1937 by Clarkson [19] as

$$
C_{\mathrm{NJ}}(X)=\sup \left\{\frac{\|x+y\|^{2}+\|x-y\|^{2}}{2\left(\|x\|^{2}+\|y\|^{2}\right)}: x, y \in X,(x, y) \neq(0,0)\right\} .
$$

We collect some properties about von Neumann-Jordan constant (see [5,20]):

(1) $1 \leqslant C_{\mathrm{NJ}}(X) \leqslant 2 ; X$ is a Hilbert space if and only if $C_{\mathrm{NJ}}(X)=1$;

(2) $X$ is uniformly non-square if and only if $C_{\mathrm{NJ}}(X)<2$;

(3) $C_{\mathrm{NJ}}(X)=C_{\mathrm{NJ}}\left(X^{*}\right)$.

\section{The Constant $L_{Y \mathrm{~J}}(\lambda, \mu, X)$}

From now on, we will consider only Banach spaces of dimension at least 2. Now, let us introduce the following key constant based on the Euler-Lagrange type identity: for $\lambda, \mu>0$

$$
L_{\mathrm{YJ}}(\lambda, \mu, X)=\sup \left\{\frac{\|\lambda x+\mu y\|^{2}+\|\mu x-\lambda y\|^{2}}{\left(\lambda^{2}+\mu^{2}\right)\left(\|x\|^{2}+\|y\|^{2}\right)}: x, y \in X,(x, y) \neq(0,0)\right\} .
$$

Proposition 1. Suppose that $X$ is a normed space. Then

$$
1 \leqslant L_{Y \mathrm{~J}}(\lambda, \mu, X) \leqslant 2
$$

Proof. Let $x \neq 0, y=0$, then clearly

$$
\frac{\|\lambda x+\mu y\|^{2}+\|\mu x-\lambda y\|^{2}}{\left(\lambda^{2}+\mu^{2}\right)\left(\|x\|^{2}+\|y\|^{2}\right)}=\frac{\|\lambda x\|^{2}+\|\mu x\|^{2}}{\left(\lambda^{2}+\mu^{2}\right)\|x\|^{2}}=1,
$$

which implies the left inequality.

To prove the right inequality:

$$
\begin{aligned}
\frac{\|\lambda x+\mu y\|^{2}+\|\mu x-\lambda y\|^{2}}{\left(\lambda^{2}+\mu^{2}\right)\left(\|x\|^{2}+\|y\|^{2}\right)} & \leqslant \frac{2\|\lambda x\|^{2}+2\|\mu y\|^{2}+2\|\mu x\|^{2}+2\|\lambda y\|^{2}}{\left(\lambda^{2}+\mu^{2}\right)\left(\|x\|^{2}+\|y\|^{2}\right)} \\
& =\frac{\left(2 \lambda^{2}+2 \mu^{2}\right)\left(\|x\|^{2}+\|y\|^{2}\right)}{\left(\lambda^{2}+\mu^{2}\right)\left(\|x\|^{2}+\|y\|^{2}\right)}=2 .
\end{aligned}
$$

This completes the proof.

Clearly, the $L_{\mathrm{YJ}}(\lambda, \mu, X)$ constant also can be rewritten as the following form:

$$
L_{\mathrm{YJ}}(\lambda, \mu, X)=\sup \left\{\frac{\|\lambda x+\mu y\|^{2}+\|\mu x-\lambda y\|^{2}}{\left(\lambda^{2}+\mu^{2}\right)\left(\|x\|^{2}+\|y\|^{2}\right)}: x, y \in X,\|x\|=1,\|y\| \leqslant 1\right\} .
$$


or equivalently

$$
L_{\mathrm{YJ}}(\lambda, \mu, X)=\sup \left\{\frac{\|\lambda x+\mu t y\|^{2}+\|\mu x-\lambda t y\|^{2}}{\left(\lambda^{2}+\mu^{2}\right)\left(1+t^{2}\right)}: x, y \in S_{X}, 0 \leqslant t \leqslant 1\right\} .
$$

\section{Proposition 2.}

$$
L_{\mathrm{YJ}}(\lambda, \mu, X)=\sup \left\{\frac{\|\lambda x+\mu y\|^{2}+\|\mu x-\lambda y\|^{2}}{2\left(\lambda^{2}+\mu^{2}\right)}:\|x\|^{2}+\|y\|^{2}=2\right\}
$$

Proof. Let us assume that $K=\sup \left\{\|\lambda x+\mu y\|^{2}+\|\mu x-\lambda y\|^{2}:\|x\|^{2}+\|y\|^{2}=2\right\}$. Clearly, then $L_{Y \mathrm{~J}}(\lambda, \mu, X) \geqslant \frac{K}{2\left(\lambda^{2}+\mu^{2}\right)}$. We shall show $L_{Y \mathrm{~J}}(\lambda, \mu, X) \leqslant \frac{K}{2\left(\lambda^{2}+\mu^{2}\right)}$. Assume that $x, y \in S_{X}$ and let $0 \leqslant t \leqslant 1$. Put

$$
u=\frac{\sqrt{2} x}{\sqrt{1+t^{2}}}, \quad v=\frac{\sqrt{2} t y}{\sqrt{1+t^{2}}} .
$$

Then $\|u\|^{2}+\|v\|^{2}=2$ and we derive

$$
\frac{\|\lambda x+\mu t y\|^{2}+\|\mu x-\lambda t y\|^{2}}{\left(\lambda^{2}+\mu^{2}\right)\left(1+t^{2}\right)}=\frac{\|\lambda u+\mu v\|^{2}+\|\mu u-\lambda v\|^{2}}{2\left(\lambda^{2}+\mu^{2}\right)} \leqslant \frac{K}{2\left(\lambda^{2}+\mu^{2}\right)},
$$

which gives $L_{Y \mathrm{~J}}(\lambda, \mu, X) \leqslant \frac{K}{2\left(\lambda^{2}+\mu^{2}\right)}$.

The next Theorem we need is a classic one, given by Jordan and von Neumann as follows when the norm is derived from an inner product.

Theorem 1 ([21]). Let $(X,\|\cdot\|)$ be a real normed linear space. Then $\|\cdot\|$ derives from an inner product if and only if the parallelogram law holds, i.e.,

$$
\|x+y\|^{2}+\|x-y\|^{2}=2\|x\|^{2}+2\|y\|^{2}
$$

for all $x, y \in X$.

Theorem 1 has some other versions where the sign of equality is replaced by the sign of inequality.

Theorem 2 ([22]). Let $(X,\|\cdot\|)$ be a real normed linear space. Then $\|\cdot\|$ derives from an inner product if and only if

$$
\|x+y\|^{2}+\|x-y\|^{2} \sim 2\|x\|^{2}+2\|y\|^{2}
$$

for all $x, y \in X$, where $\sim$ stands either for $\leqslant$ or $\geqslant$.

In article [23] the authors introduce Euler-Lagrange norms and consider Euler-Lagrange type identity as follows

$$
\|\lambda x+\mu y\|^{2}+\|\mu x-\lambda y\|^{2}=\left(\lambda^{2}+\mu^{2}\right)\left(\|x\|^{2}+\|y\|^{2}\right)
$$

for any non-negative real numbers $\lambda, \mu$ and any $x, y \in X$.

We now state the result of their subsequent articles (the relevant result is explained in [15]), which plays a vital role in our proof.

Theorem 3 ([15]). A normed space $(X,\|\cdot\|)$ is an inner product space if and only if

$$
\|\lambda x+\mu y\|^{2}+\|\mu x-\lambda y\|^{2}=\left(\lambda^{2}+\mu^{2}\right)\left(\|x\|^{2}+\|y\|^{2}\right)
$$

for any non-negative real numbers $\lambda, \mu$ and any $x, y \in X$. 
We now introduce the following Proposition inspired by Theorem 3, which is in the another type of Theorem 3.

Proposition 3. A normed space $(X,\|\cdot\|)$ is an inner product space if and only if

$$
\|\lambda x+\mu y\|^{2}+\|\mu x-\lambda y\|^{2} \leqslant\left(\lambda^{2}+\mu^{2}\right)\left(\|x\|^{2}+\|y\|^{2}\right)
$$

for any non-negative real numbers $\lambda, \mu$ and any $x, y \in X$.

Proof. Assume that $X$ is an inner product space and using Theorem 3 we see that

$$
\|\lambda x+\mu y\|^{2}+\|\mu x-\lambda y\|^{2}=\left(\lambda^{2}+\mu^{2}\right)\left(\|x\|^{2}+\|y\|^{2}\right),
$$

which implies that

$$
\|\lambda x+\mu y\|^{2}+\|\mu x-\lambda y\|^{2} \leqslant\left(\lambda^{2}+\mu^{2}\right)\left(\|x\|^{2}+\|y\|^{2}\right)
$$

for any non-negative real numbers $\lambda, \mu$ and any $x, y \in X$.

Conversely, it follows from setting $\lambda=\mu=1$ that

$$
\|x-y\|^{2}+\|x+y\|^{2} \leqslant 2\left(\|x\|^{2}+\|y\|^{2}\right),
$$

which together with Theorem 2 shows that $X$ is an inner product space.

Remark 1. Based on Theorem 2, Proposition 3 also occurs for inverse inequality.

Theorem 4. Let $X$ be a Banach space. Then $L_{Y \mathrm{~J}}(\lambda, \mu, X)=1$ if and only if $X$ is a Hilbert space.

Proof. Suppose $L_{Y \mathrm{~J}}(\lambda, \mu, X)=1$, then we have

$$
\frac{\|\lambda x+\mu y\|^{2}+\|\mu x-\lambda y\|^{2}}{\left(\lambda^{2}+\mu^{2}\right)\left(\|x\|^{2}+\|y\|^{2}\right)} \leqslant 1
$$

and hence

$$
\|\lambda x+\mu y\|^{2}+\|\mu x-\lambda y\|^{2} \leqslant\left(\lambda^{2}+\mu^{2}\right)\left(\|x\|^{2}+\|y\|^{2}\right)
$$

for any non-negative real numbers $\lambda, \mu$ and any $x, y \in X$. Thus, directly from Proposition 3 , $X$ is a Hilbert space.

For the converse, assume that $X$ is a Hilbert space, from Theorem 3 then we have

$$
\frac{\|\lambda x+\mu y\|^{2}+\|\mu x-\lambda y\|^{2}}{\left(\lambda^{2}+\mu^{2}\right)\left(\|x\|^{2}+\|y\|^{2}\right)}=1
$$

for any non-negative real numbers $\lambda, \mu$ and any $x, y \in X$. Then $L_{Y \mathrm{~J}}(\lambda, \mu, X)=1$.

Let $(X,\|\cdot\|)$ be a real or complex normed space. Suppose that the norm $\|\cdot\|$ is equivalent to a norm $\|\cdot\|_{i}$ coming from an inner product. More precisely, for $\epsilon \geqslant 0$ we have

$$
\frac{1}{1+\epsilon}\|x\|_{i} \leqslant\|x\| \leqslant(1+\epsilon)\|x\|_{i}, \quad x \in X
$$

In article [24], the author shows that if $\|\cdot\|_{i}$ is an equivalent norm coming from an inner product, then the original norm $\|\cdot\|$ satisfies an approximate parallelogram law. Following this idea, we can also establish the same results, replace approximate parallelogram law with approximate Euler-Lagrange type identity law.

Since $\|\cdot\|_{i}$ is an equivalent norm then

$$
\|\lambda x+\mu y\|^{2}+\|\mu x-\lambda y\|^{2} \leqslant(1+\epsilon)^{2}\left(\|\lambda x+\mu y\|_{i}^{2}+\|\mu x-\lambda y\|_{i}^{2}\right), x, y \in X
$$


and

$$
\frac{1}{\left(\lambda^{2}+\mu^{2}\right)\left(\|x\|^{2}+\|y\|^{2}\right)} \leqslant(1+\epsilon)^{2} \frac{1}{\left(\lambda^{2}+\mu^{2}\right)\left(\|x\|_{i}^{2}+\|y\|_{i}^{2}\right)}, x, y \in X,(x, y) \neq(0,0) .
$$

Moreover, the norm $\|\cdot\|_{i}$ satisfies the Euler-Lagrange type identity law, then we have

$$
\frac{\|\lambda x+\mu y\|^{2}+\|\mu x-\lambda y\|^{2}}{\left(\lambda^{2}+\mu^{2}\right)\left(\|x\|^{2}+\|y\|^{2}\right)} \leqslant(1+\epsilon)^{4} \frac{\|\lambda x+\mu y\|_{i}^{2}+\|\mu x-\lambda y\|_{i}^{2}}{\left(\lambda^{2}+\mu^{2}\right)\left(\|x\|_{i}^{2}+\|y\|_{i}^{2}\right)}=(1+\epsilon)^{4}
$$

for $(x, y) \neq(0,0)$.

On the other hand, we can also conclude that

$$
\frac{\|\lambda x+\mu y\|^{2}+\|\mu x-\lambda y\|^{2}}{\left(\lambda^{2}+\mu^{2}\right)\left(\|x\|^{2}+\|y\|^{2}\right)} \geqslant \frac{1}{(1+\epsilon)^{4}}
$$

for $(x, y) \neq(0,0)$. Thus we obtain

$$
\frac{1}{(1+\epsilon)^{4}} \leqslant \frac{\|\lambda x+\mu y\|^{2}+\|\mu x-\lambda y\|^{2}}{\left(\lambda^{2}+\mu^{2}\right)\left(\|x\|^{2}+\|y\|^{2}\right)} \leqslant(1+\epsilon)^{4}, x, y \in X,(x, y) \neq(0,0) .
$$

Taking $\delta:=(1+\epsilon)^{4}-1$, we can write in the form

$$
\frac{1}{1+\delta} \leqslant \frac{\|\lambda x+\mu y\|^{2}+\|\mu x-\lambda y\|^{2}}{\left(\lambda^{2}+\mu^{2}\right)\left(\|x\|^{2}+\|y\|^{2}\right)} \leqslant 1+\delta, x, y \in X,(x, y) \neq(0,0)
$$

or, equivalently,

$\left|\|\lambda x+\mu y\|^{2}+\|\mu x-\lambda y\|^{2}-\left(\lambda^{2}+\mu^{2}\right)\left(\|x\|^{2}+\|y\|^{2}\right)\right| \leqslant \delta\left(\lambda^{2}+\mu^{2}\right)\left(\|x\|^{2}+\|y\|^{2}\right), x, y \in X$.

Proposition 4. If a real or complex normed space $X$ is equivalent to an inner product space, i.e., if

$$
\frac{1}{1+\epsilon}\|x\|_{i} \leqslant\|x\| \leqslant(1+\epsilon)\|x\|_{i}, x \in X
$$

holds, then the norm satisfies the approximate Euler-Lagrange type identity law with $\delta=(1+$ $\epsilon)^{4}-1$.

Proof. It can be directly concluded from the above discussion.

Theorem 5. A Banach space $X$ with $L_{Y \mathrm{~J}}(\lambda, \mu, X)<\frac{(3 \lambda-\mu)^{2}+(\lambda+\mu)^{2}}{2\left(\lambda^{2}+\mu^{2}\right)}$ for some $\lambda, \mu>0$ is uniformly non-square.

Proof. Without loss of generality, let $\lambda \leqslant \mu$. Suppose $X$ is not uniformly non-square. $\forall 0<\delta<\lambda^{2}, \exists x, y \in S_{X}$, such that both $\|x+y\|$ and $\|x-y\|>2-\frac{\delta}{4 \lambda^{2}}$.

Step 1. Let $t_{1}=\frac{\mu}{\lambda}$, then

$$
\begin{aligned}
\left\|x+t_{1} y\right\| & =\left\|(x+y)+\left(t_{1}-1\right) y\right\| \\
& \geqslant\|x+y\|-\left\|\left(t_{1}-1\right) y\right\| \\
& \geqslant 2-\frac{\delta}{4 \lambda^{2}}-\left(t_{1}-1\right) \\
& =3-t_{1}-\frac{\delta}{4 \lambda^{2}} .
\end{aligned}
$$


So we have

$$
\|\lambda x+\mu y\| \geqslant 3 \lambda-\mu-\frac{\delta}{4 \lambda}
$$

Step 2. Let $t_{2}=\frac{\lambda}{\mu}$, then

$$
\begin{aligned}
\left\|x-t_{2} y\right\| & =\left\|(x-y)+\left(1-t_{2}\right) y\right\| \\
& \geqslant\|x-y\|-\left\|\left(1-t_{2}\right) y\right\| \\
& \geqslant 2-\frac{\delta}{4 \lambda^{2}}-\left(1-t_{2}\right) \\
& =1+t_{2}-\frac{\delta}{4 \lambda^{2}} .
\end{aligned}
$$

Thus

$$
\|\mu x-\lambda y\| \geqslant \lambda+\mu-\frac{\delta \mu}{4 \lambda^{2}}
$$

So it is easily to check

$$
\|\lambda x+\mu y\|^{2}+\|\mu x-\lambda y\|^{2} \geqslant\left(3 \lambda-\mu-\frac{\delta}{4 \lambda}\right)^{2}+\left(\lambda+\mu-\frac{\delta \mu}{4 \lambda^{2}}\right)^{2} .
$$

Since $\delta$ can be arbitrarily small, then

$$
L_{Y J}(\lambda, \mu, X) \geqslant \frac{(3 \lambda-\mu)^{2}+(\lambda+\mu)^{2}}{2\left(\lambda^{2}+\mu^{2}\right)} .
$$

This completes the proof.

A Banach space $X$ is said to be finitely representable in $E$ provided for any $\lambda>1$ and each finite-dimensional subspace $X_{1}$ of $X$, there is an isomorphism $T$ of $X_{1}$ into $E$ for which

$$
\lambda^{-1} \leqslant\|T x\| \leqslant \lambda\|x\| \text { for all } x \in X_{1} .
$$

$E$ is said to be super-reflexive ([25]) if no non-reflexive Banach space is finitely representable in $E$.

The following lemma is rather well-known, playing a key role in the geometry of Banach Spaces, which is in the same vein as to original results in [18].

Lemma 1 ([26]). Any uniformly non-square Banach space is super-reflexive.

Corollary 1. If $L_{\mathrm{YJ}}(\lambda, \mu, X)<2$ then $X$ is super-reflexive Banach space.

Proof. It follows from Theorem 5 and let $\lambda=\mu=1$, as stated.

Remark 2. Corollary 1 can also be understand in a different way. Since $L_{Y J}(\lambda, \mu, X)<2$, then for any $x, y \in S_{X}$, the following inequality is true

$$
\begin{aligned}
\min \{\|\lambda x+\mu y\|,\|\mu x-\lambda y\|\} & \leqslant\left(\frac{1}{2}\left(\|\lambda x+\mu y\|^{2}+\|\mu x-\lambda y\|^{2}\right)\right)^{\frac{1}{2}} \\
& \leqslant\left(\frac{1}{2} L_{Y J}(\lambda, \mu, X)\left(\lambda^{2}+\mu^{2}\right)\left(\|x\|^{2}+\|y\|^{2}\right)\right)^{\frac{1}{2}} .
\end{aligned}
$$

Thus we obtain

$$
\begin{aligned}
\min \left\{\left\|\frac{\lambda}{\sqrt{\lambda^{2}+\mu^{2}}} x+\frac{\mu}{\sqrt{\lambda^{2}+\mu^{2}}} y\right\|,\left\|\frac{\mu}{\sqrt{\lambda^{2}+\mu^{2}}} x-\frac{\lambda}{\sqrt{\lambda^{2}+\mu^{2}}} y\right\|\right\} & \leqslant \frac{\sqrt{2}}{2} L_{Y J}(\lambda, \mu, X)^{\frac{1}{2}}\left(\|x\|^{2}+\|y\|^{2}\right)^{\frac{1}{2}} \\
& =\sqrt{2}(1-\epsilon),
\end{aligned}
$$




$$
\begin{gathered}
\text { where } \epsilon=1-\left(\frac{L_{Y J}(\lambda, \mu, X)}{2}\right)^{\frac{1}{2}} \text {. It follows from setting } \lambda=\mu=1 \text { that } \\
\qquad \min \{\|x+y\|,\|x-y\|\} \leqslant 2(1-\epsilon),
\end{gathered}
$$

which together with Lemma 1 shows that $X$ is super-reflexive.

In the next portion, we will see that the constant $L_{Y J}(\lambda, \mu, X)$ and the weak normal structure has a nice relationship. Brodskii et al. [27] introduced some geometric concepts for the first time in 1948 as:

Definition 1. Let $K$ be a non-singleton subset of a Banach space $X$, if $K$ is closed, bounded as well as convex then $X$ holds the normal structure, whenever $r(K)<\operatorname{diam}(K)$ for every $K$, where $r(K)$ and $\operatorname{diam}(K)$ are respectively symbolized for diameter as well as for Chebyshev radius, and consequently defined mathematically as is

$$
\operatorname{diam}(K):=\sup \{\|x-y\|: x, y \in K\}
$$

and

$$
r(K):=\inf \{\sup \{\|x-y\|: y \in K\}: x \in K\}
$$

The importance of normal structure has vital applications in the field of fixed point theory with non-expansive mappings [28]. Moreover, a Banach space $X$ is said to have weak normal structure if for each weakly compact convex set $K$ in $X$ that contains more than one point has normal structure. Furthermore, if space $X$ is a reflexive Banach with normal structure, then it has the property of fixed point for nonexpansive mappings.

We begin by starting a lemma which will be our main tool.

Lemma 2 ([2]). Let $X$ be a Banach space without weak normal structure, then for any $0<\delta<1$, there exist $x_{1}, x_{2}, x_{3}$ in $S_{X}$ satisfying
(i) $x_{2}-x_{3}=a x_{1}$ with $|a-1|<\delta$;
(ii) ||$\left|x_{1}-x_{2}\left\|-1||||, x_{3}-\left(-x_{1}\right)\right\|-1\right|<\delta$; and
(iii) $\left\|\frac{x_{1}+x_{2}}{2}\right\|,\left\|\frac{x_{3}+\left(-x_{1}\right)}{2}\right\|>1-\delta$.

The geometric significance of this lemma can be interpreted as follows: if $X$ does not have weak normal structure, then there exists an inscribed hexagon in $S_{X}$ with length of each side arbitrarily closed to 1 , and with at least four sides with an arbitrarily small distance to $S_{X}$.

Theorem 6. A Banach space $X$ with $L_{Y J}(\lambda, \mu, X)<\frac{(\lambda+\mu)^{2}+(2 \mu-\lambda)^{2}}{2\left(\lambda^{2}+\mu^{2}\right)}$ for some $\lambda, \mu>0$ has weak normal structure.

Proof. Suppose $X$ does not have weak normal structure. For each $\delta>0$, let $x_{1}, x_{2}$ and $x_{3}$ in $S_{X}$ satisfying the conditions in Lemma 2 . Without loss of generality, let $\lambda \geqslant \mu$.

Step 1. Let $t=\frac{\mu}{\lambda}$. Then

$$
\begin{aligned}
\left\|x_{1}+t x_{2}\right\| & =\left\|\left(x_{1}+x_{2}\right)-(1-t) x_{2}\right\| \\
& \geqslant\left\|x_{1}+x_{2}\right\|-\left\|(1-t) x_{2}\right\| \\
& \geqslant 2-2 \delta-(1-t) \\
& =1+t-2 \delta
\end{aligned}
$$

We get

$$
\begin{aligned}
\left\|\lambda x_{1}+\mu x_{2}\right\| & =\lambda\left\|x_{1}+t x_{2}\right\| \\
& \geqslant \lambda+\mu-2 \delta \lambda .
\end{aligned}
$$


Step 2. Let $t=\frac{\mu}{\lambda}$. Then

$$
\begin{aligned}
\left\|x_{2}-t x_{1}\right\| & =\left\|x_{2}+t x_{2}-t x_{2}-t x_{1}\right\| \\
& =\left\|x_{2}+t\left(a x_{1}+x_{3}\right)-t x_{2}-t x_{1}\right\| \\
& =\left\|t\left(x_{3}-x_{1}\right)+(1-t) x_{2}+t a x_{1}\right\| \\
& \geqslant(2-2 \delta) t-1+t(1-a) \\
& \geqslant(2-2 \delta) t-\delta-1 .
\end{aligned}
$$

We get

$$
\begin{aligned}
\left\|\mu x_{1}-\lambda x_{2}\right\| & =\lambda\left\|x_{2}-t x_{1}\right\| \\
& \geqslant \lambda((2-2 \delta) t-\delta-1) .
\end{aligned}
$$

Since $\delta$ can be arbitrarily small, we deduce that

$$
L_{Y J}(\lambda, \mu, X) \geqslant \frac{(\lambda+\mu)^{2}+(2 \mu-\lambda)^{2}}{2\left(\lambda^{2}+\mu^{2}\right)} .
$$

\section{Relations with Other Geometric Constants}

In article [29], the authors show the following equivalent definition of $C_{\mathrm{NJ}}(X)$. Now, we use it to get a relation between $L_{\mathrm{YJ}}(\lambda, \mu, X)$ and $C_{\mathrm{NJ}}(X)$.

Definition 2 ([29]). Let X be a Banach space. Then

$$
C_{\mathrm{NJ}}(X)=\sup \left\{\frac{\|x+y\|^{2}+\|x-y\|^{2}}{4}:\|x\|^{2}+\|y\|^{2}=2\right\} .
$$

Proposition 5. Let X be a Banach space. Then,

$$
L_{\mathrm{YJ}}(\lambda, \mu, X) \leqslant \frac{2 \lambda^{2}}{\lambda^{2}+\mu^{2}} C_{\mathrm{NJ}}(X)+\frac{2 \sqrt{2} \lambda|\lambda-\mu|}{\lambda^{2}+\mu^{2}} \sqrt{C_{\mathrm{NJ}}(X)}+\frac{|\lambda-\mu|^{2}}{\lambda^{2}+\mu^{2}} .
$$

Proof. By use the above equivalent definition of $C_{\mathrm{NJ}}(X)$ and apply Hölder inequality, we conclude that the following estimate

$$
\begin{aligned}
& \frac{\|\lambda x+\mu y\|^{2}+\|\mu x-\lambda y\|^{2}}{\left(\lambda^{2}+\mu^{2}\right)\left(\|x\|^{2}+\|y\|^{2}\right)} \\
& \leqslant \frac{(\lambda(\|x+y\|)+|\lambda-\mu|\|y\|)^{2}+(\lambda(\|x-y\|)+|\lambda-\mu|\|x\|)^{2}}{\left(\lambda^{2}+\mu^{2}\right)\left(\|x\|^{2}+\|y\|^{2}\right)} \\
& =\frac{\lambda^{2}\left(\|x+y\|^{2}+\|x-y\|^{2}\right)+|\lambda-\mu|^{2}\left(\|x\|^{2}+\|y\|^{2}\right)+2 \lambda|\lambda-\mu|(\|x+y\|\|y\|+\|x-y\|\|x\|)}{\left(\lambda^{2}+\mu^{2}\right)\left(\|x\|^{2}+\|y\|^{2}\right)} \\
& \leqslant \frac{\lambda^{2}\left(\|x+y\|^{2}+\|x-y\|^{2}\right)+|\lambda-\mu|^{2}\left(\|x\|^{2}+\|y\|^{2}\right)+2 \lambda|\lambda-\mu| \sqrt{\|x\|^{2}+\|y\|^{2}} \sqrt{\|x+y\|^{2}+\|x-y\|^{2}}}{\left(\lambda^{2}+\mu^{2}\right)\left(\|x\|^{2}+\|y\|^{2}\right)} \\
& \leqslant \frac{2 \lambda^{2}}{\lambda^{2}+\mu^{2}} C_{\mathrm{NJ}}(X)+\frac{2 \sqrt{2} \lambda|\lambda-\mu|}{\lambda^{2}+\mu^{2}} \sqrt{C_{\mathrm{NJ}}(X)}+\frac{|\lambda-\mu|^{2}}{\lambda^{2}+\mu^{2}},
\end{aligned}
$$

which implies that the right inequality.

Example 1. Let $\lambda=1, \mu=2$, then

$$
L_{\mathrm{YJ}}(1,2, X)=\sup \left\{\frac{\|x+2 y\|^{2}+\|2 x-y\|^{2}}{5\left(\|x\|^{2}+\|y\|^{2}\right)}: x, y \in X,(x, y) \neq(0,0)\right\} .
$$


Thus from Proposition 5 we have

$$
L_{\mathrm{YJ}}(1,2, X) \leqslant \frac{2}{5} C_{\mathrm{NJ}}(X)+\frac{2 \sqrt{2}}{5} \sqrt{C_{\mathrm{NJ}}(X)}+\frac{1}{5}
$$

If $X$ is not non-square, then $C_{\mathrm{NJ}}(X)=2$ and hence $L_{Y \mathrm{~J}}(1,2, X) \leqslant \frac{9}{5}$. Furthermore, since $X$ is not non-square, it follows that there exists $x, y \in S_{X}$ such that

$$
\|x+y\|=2,\|x-y\|=2 \text {. }
$$

This means that there exists $x, y \in S_{X}$ such that

$$
\begin{aligned}
3 & \geqslant\|x+2 y\| \\
& =\|2 x+2 y-x\| \\
& \geqslant 2\|x+y\|-\|x\| \\
& =3
\end{aligned}
$$

and

$$
\begin{aligned}
3 & \geqslant\|2 x-y\| \\
& =\|2 x-2 y+y\| \\
& \geqslant 2\|x-y\|-\|y\| \\
& =3 .
\end{aligned}
$$

So it is easily to check there exists $x, y \in S_{X}$ such that

$$
\|x+2 y\|=\|2 x-y\|=3,
$$

which implies that

$$
L_{Y \mathrm{~J}}(1,2, X)=\frac{9}{5}
$$

Lemma 3 ([30]). Let $X$ be a Banach space and $J(X)$ the James constant of $X$. Then

$$
\|x+y\|^{2}+\|x-y\|^{2} \leq 4+J(X)^{2},
$$

for any $x, y \in X$ such that $\|x\|^{2}+\|y\|^{2}=2$.

Corollary 2. Let X be a Banach space. Then,

$$
L_{Y \mathrm{~J}}(\lambda, \mu, X) \leqslant \frac{\lambda^{2}}{2 \lambda^{2}+2 \mu^{2}}\left(4+J(X)^{2}\right)+\frac{\sqrt{2} \lambda|\lambda-\mu|}{\lambda^{2}+\mu^{2}} \sqrt{4+J(X)^{2}}+\frac{|\lambda-\mu|^{2}}{\lambda^{2}+\mu^{2}} .
$$

Proof. By using the same proof as Proposition 5 and combining Lemma 3 that we can easily get the result, so we omit the proof.

Remark 3. In article [30], the author gives a proof of $C_{\mathrm{NJ}}(X) \leq 1+\frac{J(X)^{2}}{4}$. From Corollary 2, we can give a new proof by letting $\lambda=\mu=1$.

Example 2. If $X$ is uniformly non-square, then

$$
L_{Y \mathrm{~J}}(\lambda, \mu, X)< \begin{cases}\frac{9 \lambda^{2}-6 \lambda \mu+\mu^{2}}{\lambda^{2}+\mu^{2}} & \text { if } \lambda>\mu ; \\ \frac{(\lambda+\mu)^{2}}{\lambda^{2}+\mu^{2}} & \text { if } \lambda<\mu ; \\ 2 & \text { if } \lambda=\mu .\end{cases}
$$


From the well-known fact, if $X$ is uniformly non-square, then $J(X)<2$. By elementary calcuation, we can easily get the thesis.

Example 3. Consider $X$ be $\mathbb{R}^{2}$ be equipped with the norm defined by

$$
\left\|\left(x_{1}, x_{2}\right)\right\|=\max \left\{\left|x_{1}\right|,\left|x_{2}\right|, \frac{\left|x_{1}\right|+\left|x_{2}\right|}{\sqrt{2}}\right\} .
$$

It is known that $J(X)=\sqrt{2}$ (see [6]). Then we have

$$
L_{\mathrm{YJ}}(\lambda, \mu, X) \leqslant \begin{cases}\frac{(4+2 \sqrt{3}) \lambda^{2}-(2 \sqrt{3}+2) \lambda \mu+\mu^{2}}{\lambda^{2}+\mu^{2}} & \text { if } \lambda>\mu ; \\ \frac{(4-2 \sqrt{3}) \lambda^{2}+(2 \sqrt{3}-2) \lambda \mu+\mu^{2}}{\lambda^{2}+\mu^{2}} & \text { if } \lambda<\mu ; \\ \frac{3}{2} & \text { if } \lambda=\mu .\end{cases}
$$

The constant $C_{Z}(X)$ was introduced by G. Zbăganu [31]:

$$
C_{Z}(X)=\sup \left\{\frac{\|x+y\|\|x-y\|}{\|x\|^{2}+\|y\|^{2}}: x, y \in X, \text { not both zero }\right\} \text {. }
$$

Next, combining $C_{\mathrm{NJ}}(X)$ we give a relationship to $L_{\mathrm{YJ}}(\lambda, \mu, X)$.

Proposition 6. Let X be a Banach space. Then,

$$
L_{Y \mathrm{~J}}(\lambda, \mu, X) \leqslant C_{\mathrm{NJ}}(X)+\frac{(\lambda+\mu)|\lambda-\mu|}{\lambda^{2}+\mu^{2}} C_{Z}(X) .
$$

Proof. Using the equality fact that

$$
\lambda x+\mu y=\frac{(\lambda+\mu)}{2}(x+y)+\frac{\lambda-\mu}{2}(x-y)
$$

and

$$
\mu x-\lambda y=\frac{(\mu-\lambda)}{2}(x+y)+\frac{\mu+\lambda}{2}(x-y)
$$

we obtain

$$
\begin{aligned}
& \frac{\|\lambda x+\mu y\|^{2}+\|\mu x-\lambda y\|^{2}}{\left(\lambda^{2}+\mu^{2}\right)\left(\|x\|^{2}+\|y\|^{2}\right)} \\
& =\frac{\left(\left\|\frac{\lambda+\mu}{2}(x+y)+\frac{\lambda-\mu}{2}(x-y)\right\|\right)^{2}+\left(\left\|\frac{\mu-\lambda}{2}(x+y)+\frac{\mu+\lambda}{2}(x-y)\right\|^{2}\right)}{\left(\lambda^{2}+\mu^{2}\right)\left(\|x\|^{2}+\|y\|^{2}\right)} \\
& \leqslant \frac{\left(\left\|\frac{\lambda+\mu}{2}(x+y)\right\|+\left\|\frac{\lambda-\mu}{2}(x-y)\right\|\right)^{2}+\left(\left\|\frac{\mu-\lambda}{2}(x+y)\right\|+\left\|\frac{\mu+\lambda}{2}(x-y)\right\|\right)^{2}}{\left(\lambda^{2}+\mu^{2}\right)\left(\|x\|^{2}+\|y\|^{2}\right)} \\
& =\frac{\left\|\frac{(\lambda+\mu)}{2}(x+y)\right\|^{2}+\left\|\frac{\lambda-\mu}{2}(x-y)\right\|^{2}+\left\|\frac{\mu-\lambda}{2}(x+y)\right\|^{2}+\left\|\frac{\mu+\lambda}{2}(x-y)\right\|^{2}+(\lambda+\mu)|\lambda-\mu|\|x+y\|\|x-y\|}{\left(\lambda^{2}+\mu^{2}\right)\left(\|x\|^{2}+\|y\|^{2}\right)} \\
& =\frac{\frac{1}{2}\left(\lambda^{2}+\mu^{2}\right)\left(\|x+y\|^{2}+\|x-y\|^{2}\right)+(\lambda+\mu)|\lambda-\mu|\|x+y\|\|x-y\|}{\left(\lambda^{2}+\mu^{2}\right)\left(\|x\|^{2}+\|y\|^{2}\right)} \\
& \leqslant C_{\mathrm{NJ}}(X)+\frac{(\lambda+\mu)|\lambda-\mu|}{\lambda^{2}+\mu^{2}} C_{Z}(X) .
\end{aligned}
$$

This completes the proof. 
Example 4. Consider $X$ be $\mathbb{R}^{2}$ be equipped with the norm defined by

$$
\left\|\left(x_{1}, x_{2}\right)\right\|=\max \left\{\left|x_{1}\right|+(\sqrt{2}-1)\left|x_{2}\right|,\left|x_{2}\right|+(\sqrt{2}-1)\left|x_{1}\right|\right\} .
$$

It is known that $C_{\mathrm{NJ}}(X)=C_{Z}(X)=4-2 \sqrt{2}$ (see [6]). Without loss of generality, let $\lambda \neq \mu$. Thus from Proposition 6 we have

$$
L_{Y \mathrm{~J}}(\lambda, \mu, X) \leqslant(4-2 \sqrt{2})\left(\frac{2 \max \left\{\lambda^{2}, \mu^{2}\right\}}{\lambda^{2}+\mu^{2}}\right) .
$$

\section{Conclusions}

Inspired by the new characterization of inner product spaces, we introduce a geometric constant $L_{Y \mathrm{~J}}(\lambda, \mu, X)$ for a Banach space $X$. The results in this paper extend the existing ones in the literature mentioned in the Introduction. It's easy to see that von NeumannJordan constant $C_{\mathrm{NJ}}(X)$ is the special case where $\lambda=\mu$, and what conditions $\lambda$ and $\mu$ satisfy, the particular constant that we get also becomes a powerful tool for studying Banach Spaces? Besides the geometric constants mentioned in the paper, what other important geometric constants are closely related to $L_{Y \mathrm{~J}}(\lambda, \mu, X)$ ? In the future, we will use the proposed constant to solve problems in Banach spaces.

Author Contributions: Writing—original draft, Q.L. and Y.L., Writing—review and editing, Q.L. and Y.L. All authors have read and agreed to the published version of the manuscript.

Funding: This work was supported by the National Natural Science Foundation of P. R. China (Nos. 11971493 and 12071491).

Institutional Review Board Statement: Not applicable.

Informed Consent Statement: Not applicable.

Data Availability Statement: Not applicable.

Acknowledgments: The authors thank anonymous referees for their remarkable comments, suggestions, and ideas that help to improve this paper.

Conflicts of Interest: The authors declare no conflict of interest.

\section{References}

1. Gao, J. On some geometric parameters in Banach spaces. J. Math. Anal. Appl. 2007, 1, 114-122. [CrossRef]

2. Gao, J.; Lau, K.S. On two classes of Banach spaces with uniform normal structure. Studia Math. 1991, 99, 41-56. [CrossRef]

3. Yang, C.; Wang, F. On estimates of the generalized Jordan-von Neumann constant of Banach spaces. J. Inequalities Pure Appl. Math. 2006, 7, 194-199.

4. Kato, M.; Maligranda, L.; Takahashi, Y. Von Neumann-Jordan constant and some geometrical constants of Banach spaces. In Nonlinear Analysis and Convex Analysis; Research Institute for Mathematical Sciences 1031; Kyoto University: Kyoto, Japan, 1998; pp. 68-74.

5. Kato, M.; Maligranda, L.; Takahashi, Y. On James Jordan-von Neumann constants and the normal structure coefficient of Banach spaces. Studia Math. 2001, 144, 275-295. [CrossRef]

6. Alonso, J.; Martin, P.; Papini, P. Wheeling around von Neumann-Jordan constant in Banach spaces. Studia Math. 2008, 188, 135-150. [CrossRef]

7. Dinarvand, M. On a generalized geometric constant and sufficient conditions for normal structure in Banach spaces. Acta Math. Sci. 2017, 37, 1209-1220. [CrossRef]

8. Gao, J. Research on normal structure in a Banach space via some parameters in its dual space. Commun. Korean Math. Soc. 2019, $34,465-475$.

9. Gao, J. On the generalized Pythagorean parameters and the applications in Banach spaces. Discrete Contin. Dyn. Syst. Ser. B 2007, 8,557-567. [CrossRef]

10. Jiménez-Melado, A.; Llorens-Fuster, E.; Saejung, S. The von Neumann-Jordan constant, weak orthogonality and normal structure in Banach spaces. Proc. Am. Math. Soc. 2006, 134, 355-364. [CrossRef]

11. Komuro, N.; Saito, K.; Tanaka, R. On the class of Banach spaces with James constant $\sqrt{2}$. Math. Nachr. 2016, 289, 1005-1020. [CrossRef] 
12. Alonso, J.; Ullán, A. Moduli in normed linear spaces and characterization of inner product spaces. Arch. Math. 1992, 59, 487-495. [CrossRef]

13. Amir, D. Characterizations of Inner Product Spaces, Operator Theory: Advances and Applications; 20 Birkhäuser Verlag: Basel, Switzerland, 1986.

14. Nikodem, K.; Páles, Z.S. Characterizations of inner product spaces by strongly convex functions. Banach J. Math. Anal. 2011, 5, 83-87. [CrossRef]

15. Moslehian, M.S.; Rassias, J.M. A characterization of inner product spaces concerning an Euler-Lagrange identity. Commun. Math. Anal. 2010, 8, 16-21.

16. Marinescu, D.S.; Monea, M.; Opincariu, M.; Stroe, M. A characterization of the inner product spaces involving trigonometry. Ann. Funct. Anal. 2013, 4, 109-113. [CrossRef]

17. Marinescu, D.S.; Monea, M.; Opincariu, M.; Stroe, M. Some equivalent characterizations of inner product spaces and their consequences. Filomat 2015, 29, 1587-1599. [CrossRef]

18. James, R.C. Uniformly non-square Banach spaces. Ann. Math. 1964, 80, 542-550. [CrossRef]

19. Clarkson, J.A. The von Neumann-Jordan constant for the Lebesgue space. Ann. Math. 1937, 38, 114-115. [CrossRef]

20. Kato, M.; Takahashi, Y. On the von Neumann-Jordan constant for Banach spaces. Proc. Am. Math. Soc. 1997, 125, 1055-1062. [CrossRef]

21. Jordan, P.; Von Neumann, J. On inner products in linear metric spaces. Ann. Math. 1935, 36, 719-723. [CrossRef]

22. Day, M.M. Some characterizations of inner-product spaces. Trans. Am. Math. Soc 1947, 62, 320-337. [CrossRef]

23. Moslehian, M.S.; Rassias, J.M. Power and Euler-Lagrange norms. Aust. J. Math. Anal. Appl. 2007, 4, 1-4.

24. Chmieliński, J. Normed spaces equivalent to inner product spaces and stability of functional equations. Aequationes Math. 2014, 87, 147-157. [CrossRef]

25. James, R.C. Super-reflexive Banach spaces. Can. J. Math. 1972, 24, 896-904. [CrossRef]

26. Pisier, G. Martingales in Banach Spaces; Cambridge University Press: Cambridge, UK, 2016; Volume 155, pp. $446-447$.

27. Brodskii, M.; Milman, D. On the center of a convex Set. Proc. Am. Math. Soc. 1948, 59, 837-840.

28. Prus, S. Geometrical background of metric fixed point theory. In Handbook of Metric Fixed Point Theory; Kirk, W.A., Sims, B., Eds.; Kluwer Academic Publishers: Dordrecht, The Netherlands, 2001; pp. 93-132.

29. Takahashi, Y.; Kato, M. On a new geometric constant related to the modulus of smoothness of a Banach space. Acta Math. Sin. 2014, 30, 1526-1538. [CrossRef]

30. Yang, C. A note on Jordan-von Neumann constant and James constant. J. Math. Anal. Appl. 2009, 357, 98-102. [CrossRef]

31. Zbăganu, G. An inequality of M. Rădulescu and S. Rădulescu which characterizes inner product spaces. Rev. Roum. Math. Pures Appl. 2001, 47, 253-257. 